\title{
USO DE SUBPRODUTOS DE CARVÃO VEGETAL NA FORMAÇÃO DO PORTA- ENXERTO LIMOEIRO 'CRAVO' EM AMBIENTE PROTEGIDO'
}

\author{
MARCELO ZANETTI², JAIRO OSVALDO CAZETTA ${ }^{3}$, DIRCEU DE MATTOS JÚNIOR ${ }^{4}$, \\ SÉRGIO ALVES DE CARVALHO ${ }^{4}$
}

\begin{abstract}
RESUMO - O trabalho teve como objetivo avaliar o efeito do uso de fino de carvão no substrato e da aplicação de extrato pirolenhoso no substrato e na planta sobre o desenvolvimento de porta-enxertos de limoeiro 'Cravo' (Citrus limonia Osbeck) cultivados em ambiente protegido. O experimento foi desenvolvido no delineamento em blocos casualisados, com análise em esquema fatorial 3 × 2 × 3 , sendo: 3 proporções de fino de carvão $(0,100$

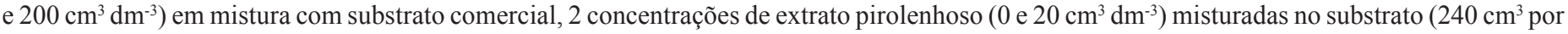
$\mathrm{dm}^{3}$ de substrato) e 3 concentrações de extrato pirolenhoso $\left(0,5 \mathrm{e} 10 \mathrm{~cm}^{3} \mathrm{dm}^{-3}\right)$ pulverizadas na parte aérea, em 4 repetições. Aos 150 e 180 dias após o plantio foram realizadas avaliações do desenvolvimento das plantas. As misturas de fino de carvão e substrato comercial contendo fino de carvão na proporção de $100 \mathrm{~cm}^{3} \mathrm{dm}^{-3}$ não influíram no desenvolvimento de porta-enxertos de limoeiro 'Cravo', porém, na proporção de $200 \mathrm{~cm}^{3} \mathrm{dm}{ }^{-3}$ prejudica o desenvolvimento dos mesmos. O umedecimento pré-plantio do substrato com solução diluída $\left(20 \mathrm{~cm}^{3} \mathrm{dm}^{-3}\right)$ de extrato pirolenhoso ou pulverização da parte aérea das plantas com soluções a 5 e $10 \mathrm{~cm}^{3} \mathrm{dm}^{-3}$ provoca redução do desenvolvimento de porta-enxertos de limoeiro 'Cravo'.
\end{abstract}

Termos para indexação: Citrus, propagação, fino de carvão, extrato pirolenhoso, substrato.

\section{THE USE OF BYPRODUCTS OF VEGETAL CHARCOAL ON GROWTH OF 'RANGPUR' LIME ROOTSTOCK UNDER SCREEN HOUSE}

\begin{abstract}
The effect of finely grounded charcoal and pyroligneous acid (PA) in mixture with a commercial growing media and PA sprayed on leaves on growth of Rangpur lime (Citrus limonia Osbeck) seedlings under screen house was studied. Treatments consisted in a combination of 3 levels of charcoal $\left(0,100\right.$ and $\left.200 \mathrm{~cm}^{3} \mathrm{dm}^{-3}\right)$ mixture on the commercial growing media, 2 rates of PA mixtured $\left(0\right.$ and $\left.20 \mathrm{~cm}^{3} \mathrm{dm}^{-3}\right)$ to a growing media $\left(240 \mathrm{~cm}^{3}\right.$ per $\mathrm{dm}^{3}$ of growing media), and 3 rates of PA $\left(0,5\right.$ and $\left.10 \mathrm{~cm}^{3} \mathrm{dm}^{-3}\right)$ sprayed on the leaves, with 4 replications. The experiment was carried out in a randomized complete block design, and analyzed as a factorial $3 \times 2 \times 3$. Growth of seedlings was evaluated at 150 and 180 days after planting. The presence of charcoal at $100 \mathrm{~cm}^{3} \mathrm{dm}^{-3}$ in the media did not influence on growth of seedlings. However, the mixture with $200 \mathrm{~cm}^{3} \mathrm{dm}^{-3} \mathrm{of}$ charcoal reduced production of total dry mass and high of the plants. The addition of PA (solution $20 \mathrm{~cm}^{3} \mathrm{dm}^{-3}$ ) to the media reduced plant growth, and sprayed (solutions 5 and $10 \mathrm{~cm}^{3} \mathrm{dm}^{-3}$ ) on the leaves reduced seedlings diameter.
\end{abstract}

Index terms: Citrus, propagation, finely grounded charcoal, pyroligneous acid, growing media.

\section{INTRODUÇÃO}

Entre as doenças que afetam a citricultura, a clorose variegada dos citros (CVC), causada pela bactéria Xylella fastidiosa, e a gomose, causada por fungos do gênero Phytophthora, são bastante importantes quando ocorrem na fase de produção de mudas. Assim, para garantir a qualidade sanitária das mudas foi instituído em 1994, pela Secretaria de Agricultura e Abastecimento do Estado de São Paulo, o Programa de Certificação de Mudas de Citros. Tais normas foram consolidadas em janeiro de 2003, com a proibição da produção, comercialização e trânsito de mudas cítricas provenientes de ambiente não protegido, neste estado (Carvalho, 2003).

Em função das mudanças no sistema de produção, há necessidade de se adequarem novas metodologias para a produção de mudas envasadas em ambiente protegido, visto que o custo de manutenção dessas estruturas é elevado. Um dos principais insumos utilizados na produção de mudas e porta-enxertos cítricos é o substrato. Este insumo deve apresentar boas características químicas e físicas e, principalmente, ser isento de plantas invasoras e patógenos prejudiciais à sanidade $\mathrm{e}$ ao vigor das mudas. A escolha dos materiais e a proporção destes nos substratos depende da sua disponibilidade e custo.

Há muito tempo utilizado no Japão e recentemente introduzido no Brasil, o fino de carvão e o extrato pirolenhoso, subprodutos obtidos da produção de carvão vegetal, são produtos promissores para a utilização na agricultura. No Brasil, a produção de carvão vegetal é uma prática bastante antiga, porém, a grande maioria se destina à obtenção apenas do carvão comercial, sem se preocupar em aproveitar os demais componentes.
O fino de carvão, obtido no processo de peneiramento na classificação do carvão vegetal, tem uma estrutura altamente porosa que, se misturado ao solo ou substrato pode aumentar a porosidade, a capacidade de retenção de água e facilitar a proliferação de microorganismos benéficos. De acordo com recomendações de Miyasaka et al. (2001), o produto pode ser utilizado na forma de pó, na granulação de 2 a $5 \mathrm{~mm}$, de preferência umedecido com uma solução de extrato pirolenhoso a $20 \mathrm{~cm}^{3} \mathrm{dm}^{-3}$ e ser aplicado no solo, na base de 500 a $700 \mathrm{~g} \mathrm{~m}^{-2}$, uma semana antes da semeadura ou plantio.

O extrato pirolenhoso, líquido obtido através da condensação da fumaça durante o processo de carbonização da madeira, é constituído de 800 a $900 \mathrm{~cm}^{3} \mathrm{dm}^{-3}$ de água e contém cerca de 200 componentes químicos diferentes, com destaque para o ácido acético $\left( \pm 80 \mathrm{~cm}^{3} \mathrm{dm}^{-3}\right)$, metanol $\left( \pm 20 \mathrm{~cm}^{3} \mathrm{dm}^{-3}\right)$ e acetona $\left( \pm 1 \mathrm{~cm}^{3} \mathrm{dm}^{-3}\right)$. Este produto diluído em água em concentração variando de $5 \mathrm{a} 20 \mathrm{~cm}^{3} \mathrm{dm}^{-3}$, quando aplicado ao solo, melhora suas propriedades físicas, químicas e biológicas, proporciona aumento da população de microorganismos benéficos, como actinomicetos e micorrizas, e favorece portanto, a absorção de nutrientes do solo pelo sistema radicular das plantas (Miyasaka et al., 2001).

O extrato pirolenhoso pode ser utilizado para diversos fins na agricultura, como "fertilizante orgânico" aplicado ao solo em arroz (Tsuzuki et al., 2000), sorgo (Esechie et al., 1998) e batata doce (Shibayama et al., 1998); desinfectante de solo (Doran, 1932), nematicida (Cuadra et al., 2000) e fungicida (Numata et al., 1994). Outros estudos mostram os efeitos benéficos no desenvolvimento radicular e produção, do extrato pirolenhoso associado ao fino de carvão aplicado ao solo como "fertilizante orgânico" em arroz (Tsuzuki et al., 1989), cana-de-

\footnotetext{
${ }^{1}$ (Trabalho 100/2003). Recebido em: 04/09/2003. Aceito para publicação: 07/11/2003. Parte da Dissertação de Mestrado do primeiro autor. Trabalho desenvolvido com o apoio da BIOCARBO Ind. Com. e Terra do paraíso LTDA.

${ }^{2}$ Eng $^{\circ}$ Agr $^{\circ}$. Pós Graduando em Produção Vegetal-FCAV/Unesp, Jaboticabal, SP. E-mail: zanettimarcelo@yahoo.com.br

${ }^{3}$ Eng $^{\circ}$. Agr ${ }^{\circ}$. Dr., Professor Adjunto do Departamento de Tecnologia - FCAV/Unesp, Via de Acesso Prof. Paulo Donato Castellane, s/n, CEP 14884-900, Jaboticabal, SP. Tel.: (16) 3209 2675. E-mail: cazetta@fcav.unesp.br

${ }^{4}$ Eng $^{\circ}$. Agr ${ }^{\circ}$. Dr., Pesquisador do APTA Citros "Sylvio Moreira" - CAPTACMS/IAC/SAA, Cordeirópolis, SP. E-mail: ddm@centrodecitricultura.br
} 
açúcar (Uddin et al., 1995), batata doce (Du et al., 1998) e melão (Du et al., 1997).

Mesmo com ação benéfica comprovada da mistura de extrato pirolenhoso e fino de carvão em diversas culturas, melhorando a produtividade, a atividade radicular e a qualidade dos produtos, nenhum estudo foi realizado com a cultura de citros em condições brasileiras. Resultados obtidos no Japão com diversas culturas indicam que, tanto o fino de carvão quanto o extrato pirolenhoso, podem ser subprodutos promissores para a produção de porta-enxertos cítricos de alta qualidade, com menor tempo de produção entre a semeadura até a repicagem.

Em função do acima exposto, este trabalho teve como objetivo avaliar o efeito da adição de fino de carvão e extrato pirolenhoso, como mistura em substrato comercial e pulverizado na parte aérea no desenvolvimento de porta-enxertos de limoeiro 'Cravo' (Citrus limonia Osbeck) cultivados em tubetes.

\section{MATERIALEMÉTODOS}

O trabalho foi desenvolvido entre maio e outubro de 2002, em um viveiro coberto por filme plástico transparente e com telado antiafídeos nas laterais, localizado no sistema de produção de mudas certificadas de citros, no Centro Avançado de Pesquisas Tecnológicas do Agronegócio de Citros "Sylvio Moreira", do Instituto Agronômico, em Cordeirópolis/SP.

$\mathrm{O}$ experimento foi instalado no delineamento de blocos casualizados e analisado no esquema fatorial $3 \times 2 \times 3$, correspondendo a: substratos contendo 3 concentrações de fino de carvão (0, 100 e 200 $\left.\mathrm{cm}^{3} \mathrm{dm}^{-3}\right)$; umedecimento pré-plantio dos substratos com 2 concentrações de extrato pirolenhoso $\left(0\right.$ e $\left.20 \mathrm{~cm}^{3} \mathrm{dm}^{-3}\right)$ e pulverização da parte aérea das plantas com 3 concentrações de extrato pirolenhoso $\left(0,5 \mathrm{e} 10 \mathrm{~cm}^{3} \mathrm{dm}^{-3}\right)$, totalizando 18 tratamentos. O experimento foi realizado com 4 repetições, sendo cada unidade experimental composta de 48 plantas com as 12 centrais compondo a área útil.

Utilizou-se sementes de limoeiro 'Cravo' ( $C$. limonia Osbeck), clone Limeira, colhidas de plantas registradas em maio de 2002, provenientes do Banco Ativo de Germoplasma do_referido Centro. As semeadura foi realizada em tubetes de plástico, em formato de cone com volume de $0,05 \mathrm{dm}^{3}$. Os recipientes foram alocados em bandejas plásticas perfuradas, contendo 96 células, fixadas sobre armação de ferro suspensa com capacidade para 12 bandejas. Para a mistura com o fino de carvão e o extrato pirolenhoso, foi utilizado o_substrato comercial Multiplant ${ }^{\circledR}$ (Terra do Paraíso Ltda: Holambra/SP) que é composto por casca de pinus compostada $\left(800 \mathrm{~cm}^{3} \mathrm{dm}^{-3}\right)$ e vermiculita expandida (200 $\mathrm{cm}^{3} \mathrm{dm}^{-3}$ ), com granulometria $<5 \mathrm{~mm}$. Após o preparo dos diferentes substratos a serem estudados, os mesmos foram adubados com Osmocote ${ }^{\circledR}$ 18-5-9 (Scotts:USA), que é um fertilizante de disponibilidade lenta (5 a 6 meses), misturado com os substratos na proporção $4 \mathrm{~g} \mathrm{dm}^{-}$ 3

Foram determinadas as características físicas do fino de carvão (FC), do substrato Multiplant@, bem como das misturas utilizadas no experimento. Os substratos avaliados foram: $\mathrm{FC}=$ Fino de carvão; $\mathrm{S}=$ substrato comercial (Multiplant $囚$ ); $\mathrm{S}+10 \% \mathrm{FC}=900 \mathrm{~cm}^{3} \mathrm{dm}^{-3} \mathrm{~S} 1+100$ $\mathrm{cm}^{3} \mathrm{dm}^{-3} \mathrm{FC} \mathrm{e} \mathrm{S}+20 \% \mathrm{FC}=800 \mathrm{~cm}^{3} \mathrm{dm}^{-3} \mathrm{~S} 1+200 \mathrm{~cm}^{3} \mathrm{dm}^{-3} \mathrm{FC}$

Determinou-se a porosidade total, o espaço de aeração e o espaço de água, usando-se o método da mesa de tensão descrito por De Boodt \& Verdonck (1972). A distribuição do tamanho dos agregados (granulometria) foi realizada submetendo-se $100 \mathrm{~g}$ de substrato, seco ao ar, a jogo de peneiras com malhas 4,00-2,00-1,00-0,50-0,25 e $0,125 \mathrm{~mm}$ acoplado a vibrador mecânico, por 3 minutos.

O fino de carvão foi misturado com enxada e o extrato pirolenhoso foi misturado ao substrato uma única vez, uma semana antes da semeadura, na forma de solução aquosa com extrato pirolenhoso a $20 \mathrm{~cm}^{3} \mathrm{dm}^{-3}$ na proporção de $240 \mathrm{~cm}^{3}$ da solução por $\mathrm{dm}^{3}$ do substrato. Os substratos sem extrato pirolenhoso foram molhados antes do enchimento da bandeja com água, na mesma proporção.
O tratamento correspondente à pulverização do extrato pirolenhoso na parte aérea iniciou-se 90 dias após o plantio (DAP), quando as plantas apresentavam aproximadamente $5 \mathrm{~cm}$ de altura. Utilizou-se uma bomba de pulverização costal e bico cone vazio com vazão de $0,5 \mathrm{dm}^{3}$-minuto ${ }^{-1} \mathrm{e}$ válvula de pressão constante $\left(1 \mathrm{kgf}-\mathrm{cm}^{-2}\right)$. As pulverizações foram realizadas semanalmente até 180 DAP, totalizando 12 aplicações e a quantidade de calda aplicada variou de acordo com o estádio de desenvolvimento da cultura, visando molhar as plantas até o ponto de escorrimento.

Avaliaram-se a altura $(\mathrm{cm})$ dos porta-enxertos, medindo-se desde a região do colo até a ápice das plantas, e o diâmetro $(\mathrm{cm})$ do caule na região do colo, aos 150 e 180 DAP. Ao final do experimento (180 DAP), quando os porta-enxertos estavam prontos para o transplante, avaliaram-se o número de folhas, a produção de massa seca da parte aérea e raízes ( $\left.\mathrm{g}_{\text {planta }}{ }^{-1}\right)$, bem como o volume do sistema radicular $\left(\mathrm{cm}^{3}\right)$, inserindo as raízes em uma proveta graduada parcialmente cheia com água destilada e medindo-se o volume de água deslocado. Os resultados obtidos foram submetidos à análise de variância pelo Teste $\mathrm{F}$ e as médias comparadas pelo teste de Tukey $(\mathrm{p}<0,05)$.

\section{RESULTADOS E DISCUSSÃO}

Os resultados da caracterização física dos materiais revelaram que, apesar do FC apresentar granulometria muito fina (aproximadamente $36 \%$ das partículas apresentando diâmetro menor que $0,125 \mathrm{~mm}$ ), a sua presença no substrato na proporção de 100 e $200 \mathrm{~cm}^{3} \mathrm{dm}^{-3}$ não modificou de forma significativa a distribuição do tamanho dos agregados das misturas. Tanto o substrato como as misturas apresentaram granulometria inferior a $4,0 \mathrm{~mm}$, com aproximadamente $60 \%$ dos agregados retidos na peneira de 1,0 $\mathrm{mm}$ (Figura 1).

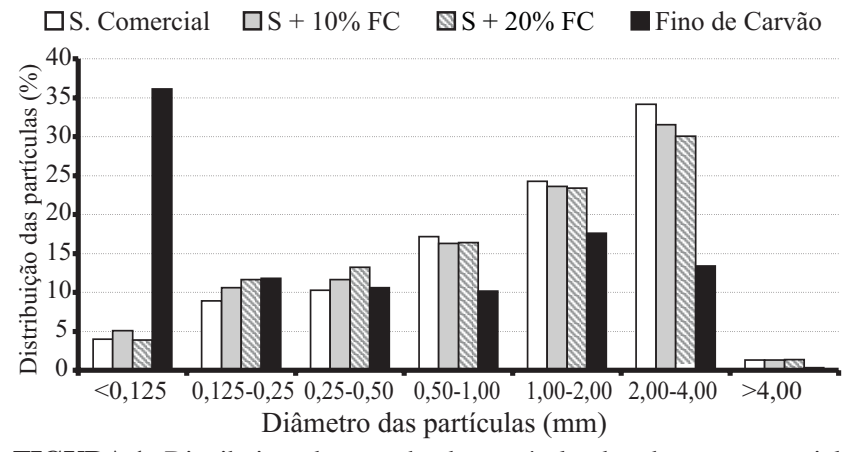

FIGURA 1 - Distribuição do tamanho das partículas do substrato comercial Multiplant@ (S), Fino de carvão (FC) e diferentes misturas utilizados para a produção de porta-enxertos de limoeiro 'Cravo'. Jaboticabal, FCAV-UNESP, 2002.

O substrato comercial, o fino de carvão e as misturas apresentaram proporções semelhantes entre sólidos (28 a 32\%) e poros (68 a 72\%). Apesar da semelhança entre as proporções de sólidos, tanto as misturas quanto o substrato comercial e o fino de carvão variaram muito no espaço de aeração (de 3 a 24\%) e na capacidade de retenção de água (de 45 a 69\%). O fino de carvão apresentou menor espaço de aeração e, conseqüentemente, maior capacidade de retenção de água; portanto, o espaço de aeração diminuiu e o espaço preenchido com água aumentou de acordo com o aumento da proporção de FC na composição do substrato (Figura 2).

Para os porta-enxertos, foram observadas diferenças significativas em relação às diferentes proporções de fino de carvão no substrato para altura aos 150 e 180 DAP e massa seca da parte aérea. O tratamento dos substratos com solução de extrato pirolenhoso ocasionou diferenças significativas para todas as variáveis analisadas 


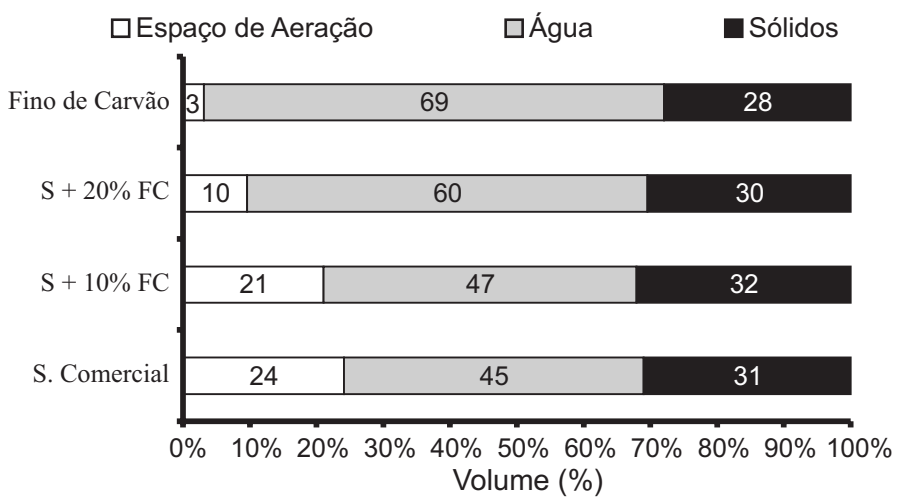

FIGURA 2 - Proporção entre os volumes de espaço de aeração, água e sólidos em Fino de carvão (FC), substrato comercial Multiplant ${ }^{\circledR}(\mathrm{S})$, mistura de $900 \mathrm{~cm}^{3} \mathrm{dm}^{-3} \mathrm{~S} 1+100 \mathrm{~cm}^{3} \mathrm{dm}^{-}$ ${ }^{3} \mathrm{FC}(\mathrm{S}+10 \% \mathrm{FC})$ e mistura de $800 \mathrm{~cm}^{3} \mathrm{dm}^{-3} \mathrm{~S} 1+200 \mathrm{~cm}^{3} \mathrm{dm}^{-}$ ${ }^{3} \mathrm{FC}(\mathrm{S}+20 \% \mathrm{FC})$, utilizados para a produção de portaenxertos de limoeiro 'Cravo'. Jaboticabal, FCAV-UNESP, 2002.

e a pulverização do mesmo na parte aérea provocou diferenças significativas apenas para diâmetro do caule aos 150 e 180 DAP. Também foram constatadas diferenças significativas para as interações FC x EP no substrato para a variável altura, em ambas as datas de avaliação, e para número de folhas e massa seca da parte aérea (Tabela 1).

Nas Tabelas 2, 3, 4 e 5 estão apresentados os dados médios para os tratamentos isolados de FC, EP no substrato e EP pulverizado na parte aérea, e interação entre FC x EP no substrato, respectivamente.

A presença de fino de carvão no substrato, na proporção de $100 \mathrm{~cm}^{3} \mathrm{dm}^{-3}$, não influiu no desenvolvimento das plantas e, portanto, pode ser considerado como alternativa para a formulação de substratos para a produção de porta-enxertos de limoeiro 'Cravo', já que esta mistura apresenta características físicas desejáveis. Porém, quando a mistura continha o FC na proporção de $200 \mathrm{~cm}^{3} \mathrm{dm}^{-3}$, o desenvolvimento dos porta-enxertos de limoeiro 'Cravo' foi prejudicado, provocando redução na altura e massa seca da parte aérea das plantas (Tabela 2).
Possivelmente os baixos valores de espaço de aeração $(9,6 \% \mathrm{v} / \mathrm{v})$ encontrados no substrato com $200 \mathrm{~cm}^{3} \mathrm{dm}^{-3}$ de fino de carvão possam ter sido o motivo do menor desempenho das plantas de limoeiro 'Cravo' (Figura 1). De acordo com Milner (2001) eAbad et al. (1989), um substrato deve conter de 20 a $30 \%$ (v/v) de espaço de aeração para que haja um bom desenvolvimento do sistema radicular e, conseqüentemente, bom crescimento da parte aérea.

A aplicação, pré-semeadura, da solução $20 \mathrm{~cm}^{3} \mathrm{dm}^{-3}$ de extrato pirolenhoso ao substrato provocou redução de todas as variáveis analisadas (Tabela 3). Tais resultados não estão em concordância com os obtidos em condições de campo por Ichikawa \& Ota (1982), que estudando a cultura do arroz antes do transplantio, observaram que a aplicação do extrato pirolenhoso no solo promoveu maior desenvolvimento da parte aérea e radicular dessas mudas, melhorando, portanto, seu desenvolvimento após o transplantio. Shirakawa et al. (1993) também relataram efeito positivo da atividade fisiológica de plantas de arroz submetidas à aplicação de extrato pirolenhoso no solo. Deve-se ressaltar, porém, que os trabalhos acima citados referem-se à utilização do EP em condições de campo e aplicação no solo e que o presente estudo foi realizado em recipiente com substrato orgânico.

A pulverização do extrato pirolenhoso na parte aérea não proporcionou diferenças significativas para nenhuma das variáveis analisadas, exceto para diâmetro do caule aos 150 e 180 DAP (Tabela 1). A pulverização com solução de EP a $5 \mathrm{~cm}^{3} \mathrm{dm}^{-3}$ provocou redução do diâmetro das plantas aos 180 DAP e a pulverização a $10 \mathrm{~cm}^{3} \mathrm{dm}^{-3}$ provocou redução no diâmetro das plantas aos 150 DAP e 180 DAP (Tabela 4).

Para a análise da interação, foi constatado que a utilização conjunta do fino de carvão e extrato pirolenhoso no substrato potencializou o efeito negativo em relação às variáveis de crescimento do limoeiro 'Cravo'. Ficou evidenciado redução da altura (150 e 180DAP), número de folhas e massa seca da parte aérea nas plantas cultivadas em substrato contendo FC na proporção de $200 \mathrm{~cm}^{3} \mathrm{dm}^{-3} \mathrm{e}$ tratado com EP, bem como redução da altura (180DAP), número de folhas e massa seca da parte aérea para as plantas cultivadas em substrato com $100 \mathrm{~cm}^{3} \mathrm{dm}^{-3} \mathrm{de}$ FC com adição de EP no substrato (Tabela $5)$.

TABELA 1 - Resultado de análise de variância de experimento sobre avaliação de porta-enxertos de limoeiro ‘Cravo’em função da aplicação de extrato pirolenhoso na parte aérea e substrato e do fino de carvão (FC) no substrato. Cordeirópolis, Centro APTA Citros "Sylvio Moreira”, 2002.

\begin{tabular}{|c|c|c|c|c|c|c|c|c|}
\hline \multirow{3}{*}{ Causas de Variação } & \multicolumn{8}{|c|}{ Variáveis } \\
\hline & \multicolumn{2}{|c|}{150 DAP } & \multicolumn{6}{|c|}{180 DAP } \\
\hline & Altura & Diâm. & Altura & Diâm. & N. folhas & MS Aérea & MS Raiz & Vol. Raiz \\
\hline Fator A (Carvão) & * & NS & $* *$ & NS & NS & $* *$ & NS & NS \\
\hline Fator B (EP Subst.) & $* *$ & $* *$ & $* *$ & $*$ & $* *$ & $* *$ & $*$ & $*$ \\
\hline Fator C (EP Foliar) & NS & $* *$ & NS & $* *$ & NS & NS & NS & NS \\
\hline Fator A x B & $* *$ & NS & $* *$ & NS & $*$ & $* *$ & NS & NS \\
\hline Fator $\mathrm{A} \times \mathrm{C}$ & NS & NS & NS & NS & NS & NS & NS & NS \\
\hline Fator $\mathrm{B} \times \mathrm{C}$ & NS & NS & NS & NS & NS & NS & NS & NS \\
\hline Fator $\mathrm{A} \times \mathrm{B} \times \mathrm{C}$ & NS & NS & NS & NS & NS & NS & NS & NS \\
\hline C.V. $(\%)$ & 6,81 & 5,00 & 5,2 & 5,60 & 3,11 & 7,89 & 13,06 & 12,90 \\
\hline
\end{tabular}

* e** significativo ao nível de $5 \%$ e $1 \%$, respectivamente, pelo teste F. DAP = Dias após plantio. NS = não significativo.

TABELA 2 - Dados médios dos tratamentos de adição de fino de carvão (FC) na composição do substrato para as variáveis de crescimento de limoeiro ‘Cravo'. Cordeirópolis, Centro APTA Citros “Sylvio Moreira”, 2002.

\begin{tabular}{|c|c|c|c|c|c|c|c|c|}
\hline \multirow{3}{*}{ Tratamentos } & \multicolumn{8}{|c|}{ Médias } \\
\hline & \multicolumn{2}{|c|}{150 DAP } & \multicolumn{6}{|c|}{180 DAP } \\
\hline & Altura & Diâm. & Altura & Diâm. & $\mathrm{N}^{\mathrm{o}}$ folhas & MS Aérea & MS Raiz & Volume Raiz \\
\hline$\left(\mathrm{cm}^{3} \mathrm{dm}^{-3}\right)$ & $(\mathrm{cm})$ & $(\mathrm{mm})$ & $(\mathrm{cm})$ & $(\mathrm{mm})$ & (unidades) & (g) & (g) & $(\mathrm{mL})$ \\
\hline 0 & $12,06 \mathrm{ab}$ & $2,08 \mathrm{a}$ & $21,25 \mathrm{a}$ & $2,52 \mathrm{a}$ & $15,46 \mathrm{a}$ & $0,990 \mathrm{a}$ & $0,317 \mathrm{a}$ & $1,44 \mathrm{a}$ \\
\hline 100 & $12,41 \mathrm{a}$ & $2,13 \mathrm{a}$ & $21,62 \mathrm{a}$ & $2,54 \mathrm{a}$ & $15,43 \mathrm{a}$ & $0,990 \mathrm{a}$ & $0,314 \mathrm{a}$ & $1,44 \mathrm{a}$ \\
\hline 200 & $11,71 \mathrm{~b}$ & $2,14 \mathrm{a}$ & $20,32 \mathrm{~b}$ & $2,54 \mathrm{a}$ & $15,20 \mathrm{a}$ & $0,919 \mathrm{~b}$ & $0,315 \mathrm{a}$ & $1,42 \mathrm{a}$ \\
\hline
\end{tabular}

Médias seguidas de mesma letra, nas colunas, não diferem entre si pelo teste de Tukey (5\%). DAP = Dias após plantio. 
TABELA 3 - Dados médios dos tratamentos da adição de extrato pirolenhoso (EP) nos substratos para as variáveis de crescimento de limoeiro 'Cravo'. Cordeirópolis, Centro APTA Citros “Sylvio Moreira”, 2002.

\begin{tabular}{|c|c|c|c|c|c|c|c|c|}
\hline \multirow{3}{*}{ Tratamentos } & \multicolumn{8}{|c|}{ Médias } \\
\hline & \multicolumn{2}{|c|}{150 DAP } & \multicolumn{6}{|c|}{180 DAP } \\
\hline & Altura & Diâm. & Altura & Diâm. & $\mathrm{N}^{\mathrm{o}}$ folhas & MS Aérea & MS Raiz & Volume Raiz \\
\hline$\left(\mathrm{cm}^{3} \mathrm{dm}^{-3}\right)$ & $(\mathrm{cm})$ & $(\mathrm{mm})$ & $(\mathrm{cm})$ & $(\mathrm{mm})$ & (unidades) & (g) & (g) & $(\mathrm{mL})$ \\
\hline 0 & $12,57 \mathrm{a}$ & $2,17 \mathrm{a}$ & $21,50 \mathrm{a}$ & $2,58 \mathrm{a}$ & $15,55 \mathrm{a}$ & $0,998 \mathrm{a}$ & $0,325 \mathrm{a}$ & $1,48 \mathrm{a}$ \\
\hline 20 & $11,54 \mathrm{~b}$ & $2,07 \mathrm{~b}$ & $20,61 \mathrm{~b}$ & $2,49 \mathrm{~b}$ & $15,17 \mathrm{~b}$ & $0,935 \mathrm{~b}$ & $0,307 \mathrm{~b}$ & $1,38 \mathrm{~b}$ \\
\hline
\end{tabular}

Médias seguidas de mesma letra, nas colunas, não diferem entre si pelo teste de Tukey (5\%). DAP = Dias após plantio.

TABELA 4 - Dados médios dos tratamentos da pulverização de extrato pirolenhoso (EP) na parte aérea das plantas para as variáveis de crescimento de limoeiro 'Cravo'. Cordeirópolis, Centro APTA Citros “Sylvio Moreira”, 2002.

\begin{tabular}{|c|c|c|c|c|c|c|c|c|}
\hline \multirow{3}{*}{ Tratamentos } & \multicolumn{8}{|c|}{ Médias } \\
\hline & \multicolumn{2}{|c|}{150 DAP } & \multicolumn{6}{|c|}{180 DAP } \\
\hline & Altura & Diâm. & Altura & Diâm. & $\mathrm{N}^{\circ}$ folhas & MS Aérea & MS Raiz & Volume Raiz \\
\hline$\left(\mathrm{cm}^{3} \mathrm{dm}^{-3}\right)$ & $(\mathrm{cm})$ & $(\mathrm{mm})$ & $(\mathrm{cm})$ & $(\mathrm{mm})$ & (unidades) & $(\mathrm{g})$ & $(\mathrm{g})$ & $(\mathrm{mL})$ \\
\hline 0 & $11,89 \mathrm{a}$ & $2,19 \mathrm{a}$ & $21,10 \mathrm{a}$ & $2,69 \mathrm{a}$ & $15,34 \mathrm{a}$ & $0,949 \mathrm{a}$ & $0,327 \mathrm{a}$ & $1,49 \mathrm{a}$ \\
\hline 5 & $12,30 \mathrm{a}$ & $2,13 \mathrm{a}$ & $21,28 \mathrm{a}$ & $2,52 \mathrm{~b}$ & $15,46 \mathrm{a}$ & $0,993 \mathrm{a}$ & $0,313 \mathrm{a}$ & $1,41 \mathrm{a}$ \\
\hline 10 & $11,98 \mathrm{a}$ & $2,04 \mathrm{~b}$ & $20,80 \mathrm{a}$ & $2,39 \mathrm{c}$ & $15,28 \mathrm{a}$ & $0,956 \mathrm{a}$ & $0,308 \mathrm{a}$ & $1,40 \mathrm{a}$ \\
\hline
\end{tabular}

Médias seguidas de mesma letra, nas colunas, não diferem entre si pelo teste de Tukey (5\%). DAP = Dias após plantio.

TABELA5 - Dados médios da interação da adição de fino de carvão e adição de extrato pirolenhoso (FC x EP) nos substratos para as variáveis altura aos 150 e 180 DAP, número de folhas e massa seca da parte aérea de limoeiro 'Cravo'. Cordeirópolis, Centro APTA Citros "Sylvio Moreira", 2002.

\begin{tabular}{|c|c|c|c|c|c|}
\hline \multirow{2}{*}{\multicolumn{2}{|c|}{ Causas de Variação }} & \multicolumn{4}{|c|}{ Médias } \\
\hline & & 150 DAP & & 180 DAP & \\
\hline $\begin{array}{l}\text { Fator A (Carvão) } \\
\left(\mathrm{cm}^{3} \mathrm{dm}^{-3}\right)\end{array}$ & $\begin{array}{l}\text { Fator B (EP Subst.) } \\
\left(\mathrm{cm}^{3} \mathrm{dm}^{-3}\right)\end{array}$ & $\begin{array}{l}\text { Altura } \\
(\mathrm{cm})\end{array}$ & $\begin{array}{l}\text { Altura } \\
(\mathrm{cm})\end{array}$ & $\begin{array}{l}\text { N. folhas } \\
\text { (unidades) }\end{array}$ & $\begin{array}{c}\text { MS Aérea } \\
(\mathrm{g})\end{array}$ \\
\hline \multicolumn{6}{|c|}{ Médias do Fator B (EP Substrato) dentro do Fator A (Carvão) } \\
\hline 0 & 0 & $12,16 \mathrm{a}$ & $21,12 \mathrm{a}$ & $15,42 \mathrm{a}$ & $0,978 \mathrm{a}$ \\
\hline 0 & 20 & $11,95 \mathrm{a}$ & $21,36 \mathrm{a}$ & $15,49 \mathrm{a}$ & $1,001 \mathrm{a}$ \\
\hline 100 & 0 & $12,89 \mathrm{a}$ & $22,07 \mathrm{a}$ & $15,67 \mathrm{a}$ & $1,031 \mathrm{a}$ \\
\hline 100 & 20 & $11,93 \mathrm{~b}$ & $21,17 \mathrm{a}$ & $15,18 \mathrm{~b}$ & $0,949 \mathrm{~b}$ \\
\hline 200 & 0 & $12,67 \mathrm{a}$ & $21,33 \mathrm{a}$ & $15,56 \mathrm{a}$ & $0,983 \mathrm{a}$ \\
\hline 200 & 20 & $10,74 \mathrm{~b}$ & $19,31 \mathrm{~b}$ & $14,85 \mathrm{~b}$ & $0,854 \mathrm{~b}$ \\
\hline \multicolumn{6}{|c|}{ Médias do Fator A (Carvão) dentro do Fator B (EP Substrato) } \\
\hline 0 & 0 & $12,16 \mathrm{a}$ & $21,12 \mathrm{a}$ & $15,42 \mathrm{a}$ & $0,978 \mathrm{a}$ \\
\hline 100 & 0 & $12,89 \mathrm{a}$ & $22,07 \mathrm{a}$ & $15,67 \mathrm{a}$ & $1,031 \mathrm{a}$ \\
\hline 200 & 0 & $12,67 \mathrm{a}$ & $21,33 \mathrm{a}$ & $15,56 \mathrm{a}$ & $0,983 \mathrm{a}$ \\
\hline 0 & 20 & $11,95 \mathrm{a}$ & $21,37 \mathrm{a}$ & $15,49 \mathrm{a}$ & $1,001 \mathrm{a}$ \\
\hline 100 & 20 & $11,93 \mathrm{a}$ & $21,17 \mathrm{a}$ & $15,18 \mathrm{ab}$ & $0,949 \mathrm{a}$ \\
\hline 200 & 20 & $10,74 \mathrm{~b}$ & $19,31 \mathrm{~b}$ & $14,85 \mathrm{~b}$ & $0,854 \mathrm{~b}$ \\
\hline
\end{tabular}

Médias seguidas de mesma letra, nas colunas e dentro dos conjuntos separados pelas linhas, não diferem entre si pelo Teste Tukey $(5 \%)$. DAP=Dias após plantio.

A utilização do extrato pirolenhoso, tanto pulverizado na parte aérea quanto incorporado ao substrato, não proporcionou resultados satisfatórios para o desenvolvimento de porta-enxertos de limoeiro 'Cravo', em ambiente protegido. Considerando a existência de vários trabalhos com resultados comprovando o efeito benéfico da utilização conjunta do fino de carvão e do extrato pirolenhoso em diversas culturas em condições de campo, são necessários novos estudos para avaliar diferentes concentrações e intervalos de aplicações em outras variedades de porta-enxerto cítricos ou em plantas adultas cultivadas no campo.

\section{CONCLUSÕES}

1) As misturas de fino de carvão e substrato comercial contendo fino de carvão na proporção de $100 \mathrm{~cm}^{3} \mathrm{dm}^{-3}$ não influíram no desenvolvimento de porta-enxertos de limoeiro 'Cravo', porém, na proporção de $200 \mathrm{~cm}^{3} \mathrm{dm}^{-3}$ prejudicam o desenvolvimento dos mesmos.

2) O umedecimento pré-plantio do substrato com solução diluída $\left(20 \mathrm{~cm}^{3} \mathrm{dm}^{-3}\right)$ de extrato pirolenhoso ou pulverização da parte aérea das plantas com soluções a 5 e $10 \mathrm{~cm}^{3} \mathrm{dm}^{-3}$, provoca redução do desenvolvimento de porta-enxertos de limoeiro 'Cravo'.

\section{REFERÊNCIAS BIBLIOGRAFICAS}

ABAD, M.; NOGUERA, V.; MATINEZ, M.M.; FORNES, F.; MARTÍNEZ, J. Physical and chemical properties of sedge peat-based media and their relation to plant growth. Acta Horticulturae, Wageningen, v. 238, p. 45-56, 1989 .

CARVALHO; S.A. Regulamentação atual da Agência de Defesa Agropecuária para a produção, estocagem, comércio, transporte e plantio de mudas cítricas no Estado de São Paulo. Laranja, Cordeirópolis, v. 24, n.1, p.199-239, 2003.

CUADRA, R.; CRUZ, X.; PERERA, E.; MARTIN, E.; DIAZ, A. Algunos compuestos naturales com efecto nematicida. Revista de Protección Vegetal, La Habana, v.24, n.15, p.31-37, 2000.

DE BOODT, M.; VERDONCK, O. The physical properties of the substrates in horticulturae. Acta Horticulturae, Wageningen, n.26, p.37-44, 1972.

DORAN, W.L. Acetic acid and pyroligneous acid in comparision with 
formaldehyde as soil desinfectants. Journal of Agricultura Research, Washington, v.44, n.7, p.571-578, 1932.

DU, H.G; MORI, E.; TERAO, H.; TSUZUKI, E. Effect of the mixture of charcoal with pyroligneous acid on shoot and root growth of sweet potato [Ipomoea batatas]. Japanese Journal of Crop Science, Bankyo-ku, Tokyo, v.67, n.2, p.149-152, 1998.

DU, H.G.; OGAWA, M.; ANDO, S.; TSUZUKI, E.; MURAYAMA, S. Effect of mixture of charcoal with pyroligneous acid on sucorose content in netted melon (Cucumis melo L. var. reticulatus Naud.) fruit. Japanese Journal of Crop Science, Bankyo-ku, Tokyo, v.66, n.3, p.369-373, 1997.

ESECHIE, H.A.; DHALIWAL, G.S.; ARORA, R.; RANDHAWA, N.S.; DHAWAN, A.K. Assessment of pyroligneous liquid as a potential organic fertilizer. In: Ecological agriculture and sustainable development, 1997, Chandigarh, India. Proceedings... Chandigarh: Center for Research in Rural and Industrial Development, 1998. v.1, p. 591-595.

ICHIKAWA, T.; OTA, Y. Effect of pyroligneous acid on the growth of rice seedlings. Japanese Journal of Crop Science, Bankyo-ku, Tokyo. v.51, p.14-17, 1982.

MILNER, L. Water and Fertlizer management in substrates. In: INTERNATIONAL CONGRESS OF CITRUS NURSERYMEN, 6 ., 2001, Ribeirão Preto. Proceedings... Ribeirão Preto: ISCN, 2001, p. 93-95.

MIYASAKA, S., OHKAWARA, T., NAGAI, K., YAZAKI, H., SAKITA, M.N. Técnicas de produção e uso do Fino de Carvão e Licor Pirolenhoso In: ENCONTRO DE PROCESSOS DE PROTEÇÃO DE
PLANTAS: Controle ecológico de pragas e doenças, 1., 2001, Botucatu. Resumo... p.161-176.

NUMATA, K.; OGAWA, T.; TANAKA, K. Effects of pyroligneous acid (wood vinegar) on the several soilborne diseases. Proceedings of the Kanto Tosan Plant Protectio Society, Omagary, v.5, n.41, p. 107-110, 1994.

SHIBAYAMA, H.; MASHIMA, K.; MITSUTOMI, M.; ARIMA, S. Effects of aplication of pyroligneous acid solution produced in Karatsu city on growth and free sugar contents of storage roots of sweet potatoes. Marine and Higland Bioscience Center Report, Phukel, v. 7, p. 15-23. 1998.

SHIRAKAWA, N.; FUKAZAWA, M.; TERADA, S. Studies on the pyroligneous acid IV. Plant physiological activities of several main components in pyroligneous acid. Japanese Journal of Crop Science, Bankyo-ku, Tokyo, v.62, p.168-189, 1993.

TSUZUKI, E.; WAKIYAMA, Y.; ETO, H.; HANDA. H. Effect of Pyroligneous Acid and Mixture of Charcoal with Pyroligneous Acid on the Growth and Yield of Rice Plant . Japan Journal Crop Science, Bankyo-ku, Tokyo, v.58, n.4, p.592-597, 1989.

TSUZUKI, E.; MORIMITSU, T.; MATSUI, T. Effect of chemical compounds in pyroligneous acid on root growth in rice plant. Japan Journal Crop Science, Bankyo-ku, Tokyo, v.66, n.4, p.15-16, 2000. UDDIN, S.M.M.; MURAYAMA, S.; ISHIMINE, Y.; TSUZUKI, E. HARADA, J. Effect of the Mixture of Charcoal with Pyroligneous Acid on dry mather production and root growth of summer planted sugarcane (Saccharum officinarum L.). Japan Journal Crop Science, Bankyo-ku, Tokyo, v.64, n.4, p.747-753, 1995. 